\title{
Evidenzbasierte Gesundheitspolitik: ein Blick in die Zukunft
}

Stefan Boes

Ausserordentlicher Professor am Seminar für Gesundheitswissenschaften und Gesundheitspolitik, Universität Luzern

Korrespondenz:

Prof. Dr. Stefan Boes

Universität Luzern

Kultur- und Sozialwissen-

schaftliche Fakultät

Frohburgstrasse 3

Postfach 4466

CH-6002 Luzern

Tel. 0412295631
Jede Reform, auch diejenige des Gesundheitswesens, ist mit Interventionen verbunden, die ein bestimmtes Ziel verfolgen. Ein Beispiel hierfür bildet die vom Bundesrat Anfang 2013 präsentierte Agenda «Gesundheit2020» [1]. Bei der Planung und Ausarbeitung solcher Interventionen braucht es ein gutes Verständnis darüber, wie die zugrundeliegenden Wirkungsmechanismen spielen und ob diese tatsächlich zielorientiert arbeiten. Dabei drängt sich eine passenderweise aus der Medizin stammende Praxis in den Vordergrund: die evidenzbasierte Gesundheitspolitik. Darunter versteht man einen politischen Entscheidungsprozess, der auf einem «gewissenhaften, ausdrücklichen und umsichtigen Gebrauch der aktuell besten Evidenz» beruht [2-3]. Durch die Zusammenarbeit von Wissenschaft und Politik soll der evidenzbasierte Ansatz dazu beitragen, Massnahmen noch wirksamer und effizienter zu gestalten, und deren Effektivität zu evaluieren.

In der Schweiz hat sich die evidenzbasierte Politik leider noch nicht als einheitliches Paradigma durchgesetzt [4], und über die «beste» Evidenz lässt sich bekanntlich diskutieren. Im Folgenden werden zwei wichtige Kriterien erläutert, die zur Beurteilung von Evidenz von Bedeutung sind. Ich beschränke mich dabei auf quantitative Evidenz; zur Qualität von qualitativen Studien siehe [5].

\section{Abbildung 1}

Forschungsdesigns und interne Validität.

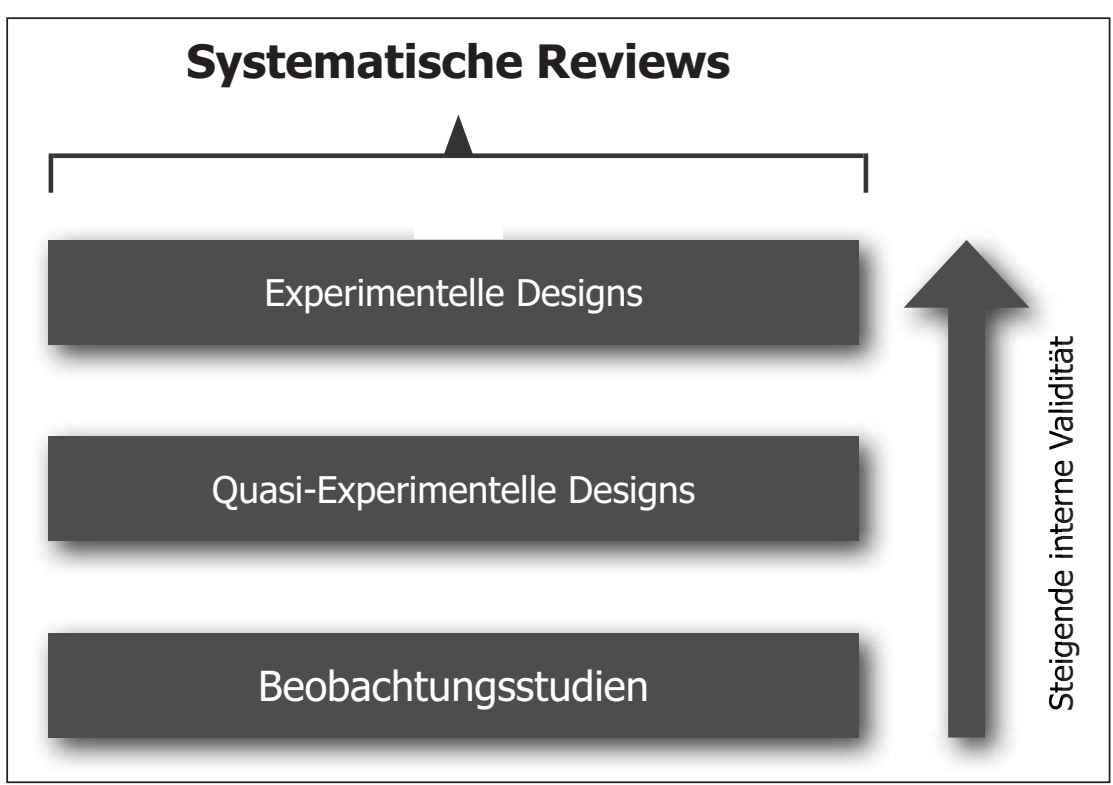

Ausgangspunkt einer jeden quantitativen Wirkungsanalyse ist die Formulierung einer kausalen Wirkungskette, d.h., was ist der Wirkungsmechanismus, der untersucht werden soll? Dieser Schritt ist eminent wichtig, da er nicht nur einen Bezug zwischen Forschung und Politik herstellt - letztlich sollte es ja die Politik sein, welche die Forschungsfragen anregt -, sondern er bildet auch das Fundament für die spätere empirische Analyse. Bei der darauffolgenden Frage nach der «besten» Evidenz wird häufig das experimentelle Ideal herangezogen. Der Grund dafür ist, dass kontrollierte und randomisierte Experimente (sogenannte RCTs - Randomized Controlled Trials) die höchste interne Validität geniessen. Dieses erste und wahrscheinlich wichtigste Kriterium bei der Beurteilung von Kausalzusammenhängen betrifft den Ausschluss möglicher Alternativerklärungen.

Während in den Naturwissenschaften Experimente weitverbreitet sind, ergeben sich bei sozialwissenschaftlichen Fragenstellungen häufig Umsetzungsprobleme, z. B. aufgrund von Kostenrestriktionen oder ethischen Bedenken. Glücklicherweise kann dort die Natur oder auch der Gesetzgeber mit seinen Regulierungen helfen, kausale Wirkungsmechanismen zu eruieren. Man spricht dabei von Quasi-Experimenten, wenn die Zuteilung der geplanten Massnahmen zwar nicht mehr zufällig erfolgt (wie bei RCTs), aber dennoch eine bestimmte Kontrolle vorliegt oder die Vergleichsgruppe geeignet gewählt werden kann [6]. Ein Beispiel ist die Änderung der Anflugregimes am Flughafen Zürich zur Beurteilung der kausalen Effekte von Fluglärm auf die Gesundheit [7].

Letztlich gibt es Studien basierend auf Beobachtungsdaten (z.B. Kohortenstudien, Fall-KontrollStudien, Quer- oder Längsschnittsanalysen), woraus jedoch nur selten kausale Schlussfolgerungen gezogen werden können, da ihre interne Validität gering ist. Aufgrunddessen sind solche Forschungsdesigns eher ungeeignet zur Beurteilung kausaler Wirkungsketten. Die Abbildung fasst die genannten Forschungsdesigns grafisch zusammen.

Als zweites Kriterium zur Beurteilung kausaler Wirkungsanalysen wird die externe Validität herangezogen. Externe Validität gibt darüber Auskunft, inwiefern die in einer konkreten Studie gefundenen Resultate verallgemeinert werden können. Dabei gilt es, insbesondere die zugrundeliegende Population (z. B. Verallgemeinerungen von der Studiengruppe 
auf die gesamte Bevölkerung) und die Sensitivität auf sich verändernde Rahmenbedingungen zu beachten.

Experimente und Quasi-Experimente geniessen also eine hohe interne Validität, d. h. innerhalb einer Studie können verlässliche Aussagen über kausale Effekte gemacht werden, aufgrund ihrer Struktur ist die externe Validität aber häufig eingeschränkt. Wünschenswert aus evidenzbasierter Sicht wäre daher die Akkumulation von mehreren (Quasi-)Experimenten, mit unterschiedlichen Personengruppen und unter variierenden Bedingungen, um dann mit Hilfe systematischer Reviews die bestmögliche Evidenz herauszuarbeiten.

\section{Für die Zukunft ist zu hoffen, dass Forschung und Politik einen noch engeren gemeinsamen Weg einschlagen.}

In diesem Sinne ist für die Zukunft zu hoffen, dass Forschung und Politik einen noch engeren gemeinsamen Weg einschlagen: Die Wissenschaft mit einer noch rigoroseren Anwendung geeigneter Forschungsdesigns zur Identifikation von Wirkungsmechanismen, und die Politik, die bei der Gestaltung von Massnahmen auf harte Evidenz aus der Wissenschaft zurückgreift. Damit würde nicht nur die Glaubwürdigkeit und Transparenz der geplanten Reformen erhöht, sondern auch sichergestellt, dass sich das schweizerische Gesundheitswesen weiterhin positiv und zum Wohle der Bevölkerung entwickelt.

\section{Literatur}

1 Bundesrat. Die gesundheitspolitischen Prioritäten des Bundesrates. Eidgenössisches Departement des Innern; 2013. www.bag.admin.ch/gesundheit2020

2 Sackett DL, Rosenberg WMC, Gray JAM, Haynes RB, Richardson WS. Evidence-based medicine: What it is and what it isn't. BMJ. 1996;312 (7023):71-2.

3 Oxman AD, Lavis JN, Lewin S, Fretheim A. Support Tools for evidence-informed health Policymaking (STP) I: What is evidence-informed policymaking? Health Research Policy and Systems. 2009;7(Suppl 1): S1.

4 Balthasar A, Rieder S. Wo ist evidenzbasierte Politik möglich? Die Verbreitung von Evaluationen auf kantonaler Ebene. In: Vatter A, Varone F, Sager F (Hrsg.). Demokratie als Leidenschaft. Planung, Entscheidung und Vollzug in der Schweizer Demokratie. Bern/Stuttgart/Wien: Haupt; 2009. S.403-29.

5 Seale C. The Quality of Qualitative Research. Thousand Oaks: Sage; 1999.

6 Shadish WR, Cook TD, Campbell DT. Experimental and quasi-experimental designs for generalized causal inference. Boston: Houghton Mifflin; 2002.

7 Boes S, Nüesch S, Stillman S. Aircraft Noise, Health, and Residential Sorting: Evidence from Two Quasi-Experiments, forthcoming in Health Economics; 2013.

\section{Schweizerischer Kongress für Gesundheitsökonomie und Gesundheitswissenschaften, \\ 25. Oktober 2013, Inselspital Bern}

Anlässlich der Durchführung des Schweizerischen Kongresses für Gesundheitsökonomie und Gesundheitswissenschaften wird in der Schriftenreihe der SGGP der Band 120 herausgegeben. Der Band unter dem Titel «Brennpunkt Gesundheitssystem» enthält Beiträge der Plenarreferenten der vergangenen 10 Kongresse. Der Beitrag von Prof. Dr. Stefan Boes ist hier als Vorabdruck erstmals publiziert. Die Beiträge umfassen ein weites gesundheitswissenschaftliches Spektrum von der Nutzenbewertung pharmakologischer Interventionen bis zu Health Impact Assessment. Am diesjährigen Kongress vom 25. Oktober werden die Professoren Katharina Janus, Stefan Boes und Martin McKee sowie Dr. Lars-Erik Holm vom Swedish National Board of Health and Welfare sich der Thematik «Gesundheitssystem 2023: Das
Gute messbar und besser machen» annehmen. Erstmals findet am Nachmittag das Zukunftsforum Gesundheit statt. Im Mittelpunkt stehen hier die Referate «E-Health - die Zukunft der Gesundheitssysteme ist digital» und «healthtalkonline der emanzipierte Patient als Herausforderung der Zukunft». Der Kongress schliesst mit einer Diskussion zum Kongressthema und der Vergabe des Gesundheitsökonomiepreises (Preisstifter: MSD). Kongresssprachen sind Deutsch und Englisch. Traditionell findet der Auftakt zum Kongress im Widder Hotel in Zürich statt. An der Vorabendveranstaltung referiert Professorin Katharina Janus zum Thema «Gesundheitsmanagement im Dialog zwischen Fiktion und Realität». Anmeldung und Information via www.skgg.ch 